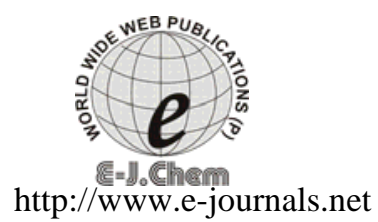

ISSN: 0973-4945; CODEN ECJHAO

E-Journal of Chemistry

2011, 8(S1), S266-S270

\title{
Trifluoroacetic Acid as an Effective Catalyst for Biginelli Reaction: One-Pot, Three-Component Synthesis of 3,4-Dihydropyrimidin-2(1H)-ones (and-Thiones)
}

\author{
MOHAMMAD R. MOHAMMADIZADEH* ${ }^{*}$ and NEDA FIROOZI
}

Department of Chemistry, Faculty of Sciences

Persian Gulf University, Bushehr 75169, Iran

mrmohamadizadeh@pgu.ac.ir

Received 11 March 2011; Accepted 23 May 2011

\begin{abstract}
Trifluoroacetic acid (TFA) is introduced as an effective catalyst for promotion of Biginelli reaction. 3,4-Dihydropyrimidin-2(1H)-one (-thione) derivatives (DHPMs) were simply and efficiently prepared using TFA catalyzed one-pot, three-component condensation $\beta$-dicarbonyl compounds, aldehydes and urea (thio).
\end{abstract}

Keywords: Biginelli reaction, TFA, 3,4-Dihydropyrimidinones, $\beta$-Dicarbonyl compounds

\section{Introduction}

Recently, DHPM and their derivatives have attracted considerable interest because they exhibit promising activities as calcium channel blockers, antihypertensive agents and $\alpha-1 \mathrm{a}-$ antagonists and neuropeptide Y (NPY) antagonists ${ }^{1}$. Furthermore, several bioactive isolated marine alkaloids were also found to contain a 2-amino-1,4-dihydropyrimidinone5-carboxylate core ${ }^{2}$. Most notably among them are the batzalladine alkaloids, which have been found to be potent HIV-gp-120-CD4 inhibitors ${ }^{3}$. Their derivatives also exhibit a wide spectrum of biological effects including antifungal, antiviral, anticancer, antibacterial, antiinflammatory and antihypertensive effects ${ }^{4}$.

The three-component cyclocondensation reaction constituting aldehyde, $\beta$-ketoester, and urea in an acidic medium, which was originally reported by Biginalli ${ }^{5 a}$, is the earliest and most effective effort to formation of DHPMs. The multi-component nature of the procedure together with the existence of a wide variety of commercially available $\beta$ dicarbonyls and aldehydes makes this reaction an ideal candidate for the combinatorial synthesis technology ${ }^{5 \mathrm{~b}}$. Although, Biginelli-type dihydropyrimidinones synthesis could be done without catalyst, but all such reported conditions used of microwave ovens ${ }^{6}$, 
or classical heating ${ }^{7}$, to reach high temperatures for promotion of reactions at acceptable rates. For this reason and due to the importance of the Biginelli reaction products, much work on improving the yields and reaction conditions has been actively pursued. Thus, in the past ten years, many improved methods, including enantioselective versions, have been exploited and till about 400 papers were correspondingly published ${ }^{8}$. In these advanced methods, Lewis or Brønsted acids were mainly used as catalyst under milder conditions with much better results compared to the results obtained by employing traditional conditions. On the other hand, this condensation was found to be equally effective when Lewis acids were replaced by a strong Brönsted base $(\mathrm{KOH})$, but in this case the reaction involves two steps ${ }^{9}$. While some of the catalysts are really very fascinating from a synthetic chemist's point, most are exotic, expensive, complex, unavailable, harmful and even ineffective in the absence of acid additives.

Trifluoroacetic acid (TFA) is the simplest perfluorinated carboxylic acid which is commercially available. TFA is a versatile solvent for acid stable compounds in NMR spectroscopy and also used as a calibrant in mass spectrometry. Furthermore, because of its interesting properties, such as low toxicity, solubility in water and organic solvents, and strength, TFA is considered to be a special reagent for highly sensitive microsequencing of proteins $^{10}$, as well as a special catalyst for promotion of numerous organic reactions ${ }^{11}$.

\section{Experimental}

TFA $(0.04 \mathrm{mmol})$ was added to a mixture of dicarbonyl compound 1 or 4 (1 mmol), benzaldehyde or appropriately substituted corresponding benzaldehyde derivatives 2 $(1 \mathrm{mmol})$ and either urea or thiourea $(1.1 \mathrm{mmol})$ in acetonitrile $(4 \mathrm{mmL})$ and the reaction mixture was heated with stirring at $70{ }^{\circ} \mathrm{C}$ for appropriate time. Then the reaction mixture was allowed to cool to room temperature, the resulting solid was filtered off and washed with $10 \mathrm{~mL}$ of hot water. Almost resulting products have sufficient purity, but more purification if necessary, can access by recrystallization of the products from ethanol.

5-Ethoxycarbonyl-6-methyl-4-(4-chlorophenyl)-3,4-dihydropyrimidin-2(1H)-one (3b)

${ }^{1} \mathrm{H}$ NMR $\left(\mathrm{CDCl}_{3}, 300 \mathrm{MHz}\right) 1.20$ (t, J = 7.1 Hz, 3H, $\mathrm{CH}_{3}$ ), 2.20 (s, 3H, $\mathrm{CH}_{3}$ ), 3.94 (q, J = 7.1 $\mathrm{Hz}, 2 \mathrm{H}, \mathrm{CH}_{2}$ ), 5.14 (s, 1H, CH), 7.35-7.25 (m, 4H, arom), 7.76 (s, 1H, NH), 9.24 (s, 1H, NH).

5-Ethoxycarbonyl-6-methyl-4-phenyl-3,4-dihydropyrimidin-2(1H)-thione (3e)

${ }^{1} \mathrm{H}$ NMR ( $\left.\mathrm{CDCl}_{3}, 300 \mathrm{MHz}\right) 1.17$ (t, 3H, J= $7.0 \mathrm{~Hz}, \mathrm{CH}_{3}$ ), 2.25 (s, 3H, $\mathrm{CH}_{3}$ ), 4.10 (q, 2H, J= 7.0 $\mathrm{Hz}, \mathrm{CH}_{2}$ ), 5.20 (s, 1H, CH), 7.35-7.19 (m, 5H, arom), 9.60 (s, 1H, NH), 10.25 (br s, 1H, NH).

\section{5-Methoxycarbonyl-6-methyl-4-(4-chlorophenyl)-3,4-dihydropyrimidin-2(1H)-one (3l)}

${ }^{1} \mathrm{H}$ NMR $\left(\mathrm{CDCl}_{3}, 300 \mathrm{MHz}\right) 2.24$ (s, 3H, $\left.\mathrm{CH}_{3}\right), 3.53$ (s, 3H, $\left.\mathrm{CH}_{3}\right), 5.14$ (s, 1H, CH), 7.417.22 (m, 4H, arom), 7.74 (s, 1H, NH), 9.27 (s, 1H, NH).

Ethyl 6-ethoxycarbonylmethyl-4-phenyl-2-oxo-1,2,3,4-tetrahydropyrimidine-5carboxylate (5a)

${ }^{1} \mathrm{H}$ NMR $\left(\mathrm{CDCl}_{3}, 300 \mathrm{MHz}\right) 1.14$ (t, 3H, J= 7.4 Hz, CH $\left.\mathrm{CH}_{3}\right), 1.26$ (t, 3H, J= 7.0 Hz, $\left.\mathrm{CH}_{3}\right), 3.67$ (d, $1 \mathrm{H}, \mathrm{J}=16.8 \mathrm{~Hz}, \mathrm{CH}_{2}$ ), 3.94 (d, $1 \mathrm{H}, \mathrm{J}=16.8 \mathrm{~Hz}, \mathrm{CH}_{2}$ ), 4.06 (q, J= 7.4 Hz, $\mathrm{CH}_{2}$ ), 4.18 (q, 2H, $\left.\mathrm{J}=7.0, \mathrm{CH}_{2}\right), 5.43$ (s, 1H, CH), $6.02(\mathrm{~s}, 1 \mathrm{H}, \mathrm{NH}),(7.28-7.41$ (m, 5H, arom), 8.91 (s, 1H, NH).

Ethyl 6-ethoxycarbonylmethyl-4-(2-bromophenyl)-2-oxo-1,2,3,4-tetrahydropyrimidine -5-carboxylate (5d)

${ }^{1} \mathrm{H}$ NMR $\left(\mathrm{CDCl}_{3}, 300 \mathrm{MHz}\right) 1.50$ (t, 3H, J= 7.2 Hz, CH ), 1.45 (t, 3H, J= 7.1 Hz, $\mathrm{CH}_{3}$ ), 3.43 (d, $\left.1 \mathrm{H}, \mathrm{J}=16.8 \mathrm{~Hz}, \mathrm{CH}_{2}\right), 4.33$ (d, 1H, J= $\left.16.8 \mathrm{~Hz}, \mathrm{CH}_{2}\right), 4.28\left(\mathrm{q}, 2 \mathrm{H}, \mathrm{J}=7.2 \mathrm{~Hz}, \mathrm{CH}_{2}\right), 4.41$ (q, 2H, $\mathrm{J}=7.1, \mathrm{CH}_{2}$ ), 5.21 (s, 1H, CH), 7.20 (s, 1H, NH), 7.02-7.61 (m, 5H, arom), 8.79 (s, 1H, NH). 
Ethyl 6-ethoxycarbonylmethyl-4-(4-chlorophenyl)-2-oxo-1,2,3,4-tetrahydropyrimidine -5-carboxylate (5e)

${ }^{1} \mathrm{H}$ NMR $\left(\mathrm{CDCl}_{3}, 300 \mathrm{MHz}\right) 1.05$ (t, 3H, J= 7.1 Hz, CH $\mathrm{CH}_{3}, 1.18$ (t, 3H, J=7.0 Hz, $\left.\mathrm{CH}_{3}\right), 3.62$ (d, $1 \mathrm{H}, \mathrm{J}=16.9 \mathrm{~Hz}, \mathrm{CH}_{2}$ ), 3.81 (d, $1 \mathrm{H}, \mathrm{J}=16.9 \mathrm{~Hz}, \mathrm{CH}_{2}$ ), 3.94 (q, $2 \mathrm{H}, \mathrm{J}=7.2 \mathrm{~Hz}, \mathrm{CH}_{2}$ ), 4.10 (q, 2H, J= 7.1, $\mathrm{CH}_{2}$ ), 5.17 (s, 1H, CH), 7.34 (d, J= 8.4 Hz, arom), 7.41 (d, J= 8.4 Hz, arom), 7.84 (s, 1H, NH), 9.33 (s, 1H, NH).

\section{Results and Discussion}

In continuing of our interest on synthesis of nitrogen containing heterocycles of potentially biologically valuables ${ }^{12}$ and as a part of our ongoing programs on studding of catalytic ability of TFA ${ }^{11 a}$, here we introduced TFA as a new inexpensive, completely soluble in water and organic solvents, and easily available catalyst for Biginelli-type synthesis of 3,4-dihydropyrimidin-2(1H)-ones (-thiones) 3 via one-pot three-component condensation of $\beta$-dicarbonyls 1, aldehydes 2 and urea or thiourea (Scheme 1).

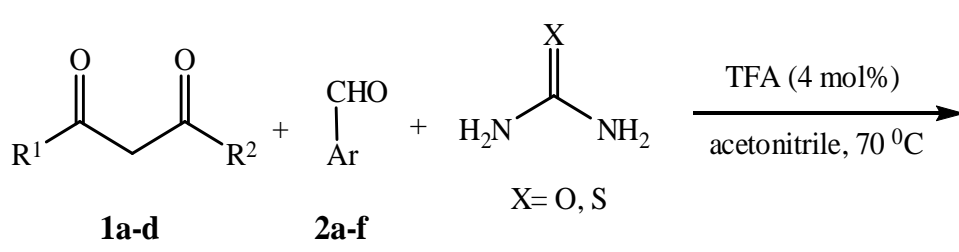<smiles>[R]C(=O)C1=C([R])NC([X])NC1[Al]</smiles>

Scheme 1. TFA-catalyzed Biginelli-type synthesis of 3,4-dihydropyrimidin-2(1H)-ones (-thiones) 3a-o

Our first finding was that the reaction of benzaldehyde and urea with ethyl acetoacetate in the presence of a catalytic amount of TFA in refluxing acetonitrile afforded the desired DHPM (3a) in 80\% yield. The procedure was optimized by varying reaction temperature, reaction time, reagents molar ratio, and solvent for this reaction. After extensive screening, we found the optimized best yields and time profiles were obtained with a molar ratio of 0.04:1:1:1.1 of TFA, dicarbonyls, aldehydes, and urea, respectively, in acetonitrile at $70{ }^{\circ} \mathrm{C}$, which furnished the corresponding DHPM (3a) in 93\% yield within 30 minutes.

Using this optimized reaction conditions, a broad range of structurally diverse 1,3-dicarbonyl compounds, aldehydes and urea are subjected under this procedure to produce the corresponding DHPMs. Thiourea also works well to give the corresponding thio derivatives which are also of much interest with regard to biological activity ${ }^{7}$. The results are summarized in Table 1. Some physical properties and NMR characterization of various selected DHPMs (3) are given. All the other products included in Table 1 were also characterized by ${ }^{1} \mathrm{H}$ NMR and ${ }^{13} \mathrm{C}$ NMR and/or by comparison with authentic samples.

Recently, we report same Biginelli-like one-pot three component procedure for preparation of 6-ethoxycarbonyldihydropyrimidinone derivatives (5a-e) using diethyl 3oxoglutarate instead of simple ordinary dicarbonyls in the presence of $p$-toluenesulfonic acid as catalyst (Scheme 2$)^{13}$. The reported procedure was very simple and effective and reviewed more recently by other researchers ${ }^{14}$. Our observation in this work (Table 2) supports the effectiveness of TFA as catalyst for preparation of the attractive 6-ethoxycarbonyl-dihydropyrimidinones (5a-e). 
Table 1. Synthesis of 3,4-dihydropyrimidin-2(1H)-ones and thiones (3a-q) in the presence of TFA

\begin{tabular}{cccccccc}
\hline $3^{\text {a,b }}$ & $\mathrm{R}^{1}$ & $\mathrm{R}^{2}$ & $\mathrm{Ar}$ & $\mathrm{X}$ & Time, & Yield $^{\mathrm{c}}$ & $\mathrm{Mp}^{[\mathrm{Ref} .]}$ \\
\hline $\mathbf{a}$ & $\mathrm{Et}$ & $\mathrm{Me}$ & $\mathrm{Ph}$ & $\mathrm{O}$ & 0.5 & 88 & $202-203^{[16]}$ \\
$\mathbf{b}$ & $\mathrm{Et}$ & $\mathrm{Me}$ & $4-\mathrm{Cl}-\mathrm{Ph}$ & $\mathrm{O}$ & 1.5 & 85 & $207-209^{[15]}$ \\
$\mathbf{c}$ & $\mathrm{Et}$ & $\mathrm{Me}$ & $4-\mathrm{Me}-\mathrm{Ph}$ & $\mathrm{O}$ & 1 & 92 & $1701-173^{[15]}$ \\
$\mathbf{d}$ & $\mathrm{Et}$ & $\mathrm{Me}$ & $4-\mathrm{MeO}-\mathrm{Ph}$ & $\mathrm{O}$ & 1 & 92 & $198-201^{[15]}$ \\
$\mathbf{e}$ & $\mathrm{Et}$ & $\mathrm{Me}$ & $2-\mathrm{O}_{2} \mathrm{~N}-\mathrm{Ph}$ & $\mathrm{O}$ & 2.1 & 90 & $217-218^{[16]}$ \\
$\mathbf{f}$ & $\mathrm{Et}$ & $\mathrm{Me}$ & $4-\mathrm{O}_{2} \mathrm{~N}-\mathrm{Ph}$ & $\mathrm{O}$ & 2 & 95 & $207-208^{[16]}$ \\
$\mathbf{g}$ & $\mathrm{Et}$ & $\mathrm{Me}$ & $4-\mathrm{F}-\mathrm{Ph}$ & $\mathrm{O}$ & 1.5 & 83 & $186-188^{[16]}$ \\
$\mathbf{h}$ & $\mathrm{Et}$ & $\mathrm{Me}$ & $4-\mathrm{Me}_{2} \mathrm{~N}-\mathrm{Ph}$ & $\mathrm{O}$ & 1.3 & 90 & $251-253^{[15]}$ \\
$\mathbf{i}$ & $\mathrm{Et}$ & $\mathrm{Me}$ & $\mathrm{Ph}$ & $\mathrm{S}$ & 1.5 & 79 & $205-207^{[8]}$ \\
$\mathbf{j}$ & $\mathrm{Et}$ & $\mathrm{Me}$ & $4-\mathrm{Me}-\mathrm{Ph}$ & $\mathrm{S}$ & 1 & 77 & $193-194^{[15]}$ \\
$\mathbf{k}$ & $\mathrm{Et}$ & $\mathrm{Me}$ & $4-\mathrm{Me} \mathrm{e}_{2} \mathrm{~N}-\mathrm{Ph}$ & $\mathrm{S}$ & 1.2 & 78 & $207-209^{[15]}$ \\
$\mathbf{l}$ & $\mathrm{Me}$ & $\mathrm{Me}$ & $\mathrm{Ph}$ & $\mathrm{O}$ & 1 & 83 & $210-212^{[17]}$ \\
$\mathbf{m}$ & $\mathrm{Me}$ & $\mathrm{Me}$ & $4-\mathrm{Me}-\mathrm{Ph}$ & $\mathrm{O}$ & 1.2 & 90 & $209-211^{[17]}$ \\
$\mathbf{n}$ & $\mathrm{Me}$ & $\mathrm{Me}$ & $4-\mathrm{Cl}-\mathrm{Ph}$ & $\mathrm{O}$ & 1 & 85 & $208-209^{[17]}$ \\
$\mathbf{o}$ & $\mathrm{Me}$ & $\mathrm{Me}$ & $4-\mathrm{MeO}-\mathrm{Ph}$ & $\mathrm{O}$ & 1.1 & 91 & $193-195^{[17]}$ \\
\hline
\end{tabular}

${ }^{a}$ Diketone/aldehydes/urea (thio)/TFA: (1:1:1.1:0.02). ${ }^{b}$ All products are known compounds and their structures were characterized by their ${ }^{l} H$ NMR and comparison of their physical properties with reported data. ${ }^{c}$ All referred to isolated pure products

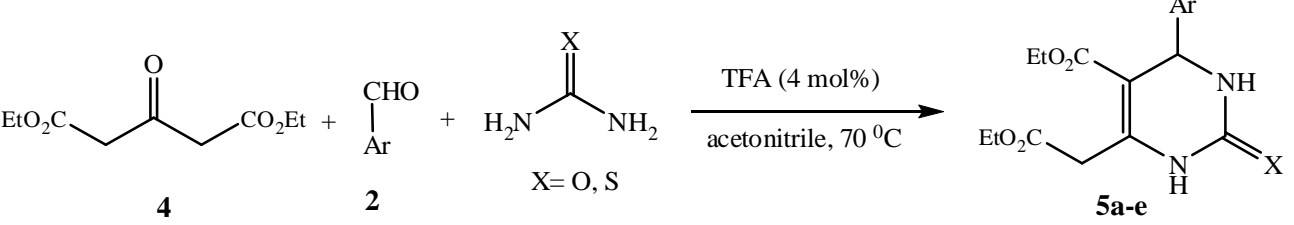

Scheme 2. TFA-catalyzed Biginelli-type synthesis of 6-ethoxycarbonyldihydropyrimidinones (5a-e)

Table 2. Physical constants of the 6-ethoxycarbonyldihydropyrimidinones (5a-e)

\begin{tabular}{cccccccccc}
\hline $5^{\mathrm{a}}$ & $\mathrm{Ar}$ & $\begin{array}{c}\text { Time, } \\
\mathrm{h}\end{array}$ & $\begin{array}{c}\text { Yield } \\
\%^{\mathrm{b}}\end{array}$ & $\begin{array}{c}\mathrm{M} . \mathrm{P} ., \\
{ }^{\circ} \mathrm{C}\end{array}$ & $\mathbf{5}^{\mathbf{a}}$ & $\mathrm{Ar}$ & $\begin{array}{c}\text { Time, } \\
\mathrm{h}\end{array}$ & $\begin{array}{c}\text { Yield } \\
\%^{\mathrm{b}}\end{array}$ & $\begin{array}{c}\text { M.P., } \\
{ }^{\circ} \mathrm{C}\end{array}$ \\
\hline $\mathbf{a}$ & $\mathrm{Ph}$ & 2 & 84 & $177-178$ & $\mathbf{d}$ & $2-\mathrm{Br}-\mathrm{Ph}$ & 2.2 & 86 & $155-156$ \\
$\mathbf{b}$ & $3-\mathrm{O}_{2} \mathrm{~N}-\mathrm{Ph}$ & 2 & 79 & $148-151$ & $\mathbf{e}$ & $4-\mathrm{Cl}-\mathrm{Ph}$ & 2 & 92 & $136-138$ \\
$\mathbf{c}$ & $4-\mathrm{Me}-\mathrm{Ph}$ & 2.2 & 75 & $133-135$ & & & & & \\
\hline
\end{tabular}

${ }^{a}$ Same conditions as Table 1 were used. ${ }^{b}$ Referred to the isolated pure products. ${ }^{c}$ Products were characterized by comparison of their physical and spectroscopic data with previously reported ones ${ }^{13}$

\section{Conclusion}

We have developed a simple and efficient method for the synthesis of dihydropyrimidinones using a catalytic amount of TFA in acetonitrile at $70{ }^{\circ} \mathrm{C}$. Mild reaction conditions, high yields of the products, ease of work-up, compatibility with various functional groups, are all characteristics that make the present method significant addition to the already existing methodologies for heterocyclics synthesis.

\section{Acknowledgment}

The authors thank the Research Committee of Persian Gulf University for financial support of this work. 


\section{References}

1 Rovnyak G C, Kimball S D, Beyer B, Cucinotta G, Dimarco J D, Gougoutas J, Hedberg A, Malley M, McCarthy J P, Zhang R and Moreland S, J Med Chem., 1995, 38, 119-129.

$2 \quad$ Snider B B and Shi Z, J Org Chem., 1993, 58, 3828-3839.

3 (a) Patil A D, Kumar N V, Kokke W C, Bean M F, Freyer A J, De Brosse C, Mai S, Truneh A, Faulkner D J, Carte B, Breen A L, Hertzberg R P, Johnson R K, Westley J W, Potts B C M, J Org Chem., 1995, 60, 1182; (b) Rao R A V, Gurjar M K and Vasudevan J, J Chem Soc Chem Commun., 1995, 13, 1369.

$4 \quad$ Kappe C O, Eur J Med Chem., 2004, 39, 777-783.

5 Biginelli P, Pure Appl Chem., 2004, 76, 1017-1024.

6 (a) Mobinikhaledi A, Foroughifar N and Fathinejad H Jirandehi, Phosphorus, Sulfur and Silicon, 2004, 179, 2259-2263; (b) Kidwai M, Saxena S, Mohan R and Venkataramanan R, J Chem Soc., Perkin Trans. 1, 2002, 1845-1846; (c) Shanmugam P, Sabastein C and Perumal P T, Indian J Chem B, 2004, 43, 135; (d) Legeay J-C, Eynde J J V and Bazureau J P, Tetrahedron, 2005, 61, 12386-12397.

$7 \quad$ Ranu B C, Hajra A and Dey S S, Org Process Res Develop., 2002, 6, 817.

8 Kolosov M A, Orlov V D, Beloborodov D A and Dotsenko V V, Mol Divers, 2009, 13, 5 and references sited therein.

9 Anatoly D S, Ekaterina A K, Natali V S and Aleksei Y K, Molecules, 1998, 3, 100.

10 Eidman K F and Nichols P J, Trifluoroacetic acid; In Encyclopedia of Reagents for Organic Synthesis; L. Paquette (Ed.); Wiley: New York, 2004.

11 (a) Mohammadizadeh M R, Hasaninejad A and Bahramzadeh M, Synth Cmmun., 2009, 39, 3232; (b) Fletcher S and Gunning P T, Tetrahedron Lett., 2008, 49, 48174819; (c) Wu, Y-C, Liu L, Liu Y-L, Wang D and Chen Y J, J Org Chem., 2007, 72(24), 9383-9386.

12 (a) Azizian J, Mohammadizadeh M R, Zomorodbakhsh S, Mohammad A A and Karimi A R Arkivoc, 2007, 15, 25; (b) Azizian J, Mohammadizadeh M R, Teimouri F, Mohammadi A A, Karimi A R, Synth Commun., 2006, 36, 3631.

13 Azizian J, Mohammad A A, Kohshari M, Karimi A R and Mohammadizadeh M R, $J$ Heterocycl Chem., 2007, 44, 455.

14 Svetlık J, Veizerova L and Kettmann V, Tetrahedron Lett., 2008, 49, 3520.

15 Jing X, Li Z, Pan X, Shi Y and Yan C, J Iran Chem Soc., 2009, 6(3), 514-518.

16 Ahmed B, Khan R A and Keshari M, Tetrahedron Lett., 2009, 50, $2889-2892$.

17 Yu Y, Liu D, Liu C and Luo G, Bioorg Med Chem Lett., 2007, 17, 3508. 


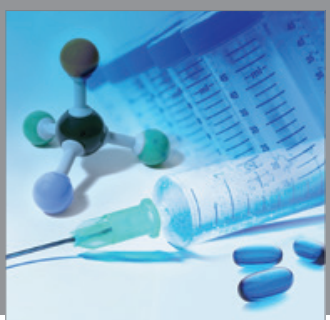

International Journal of

Medicinal Chemistry

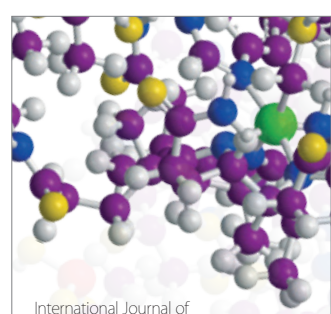

Carbohydrate Chemistry

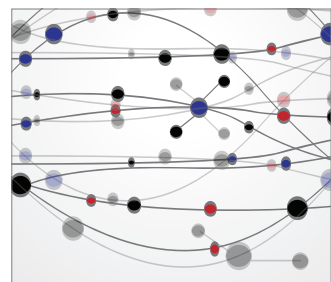

The Scientific World Journal
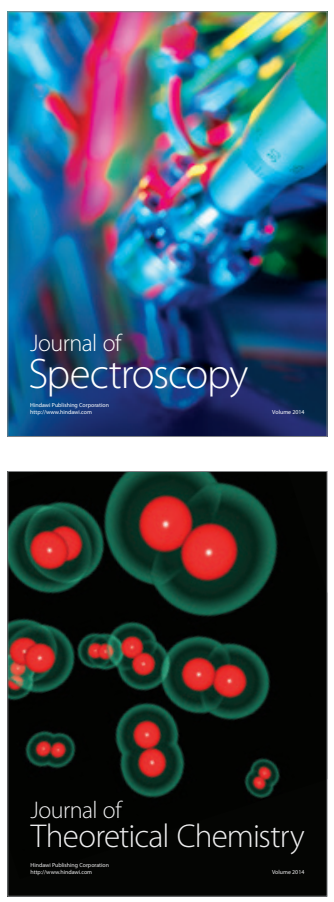
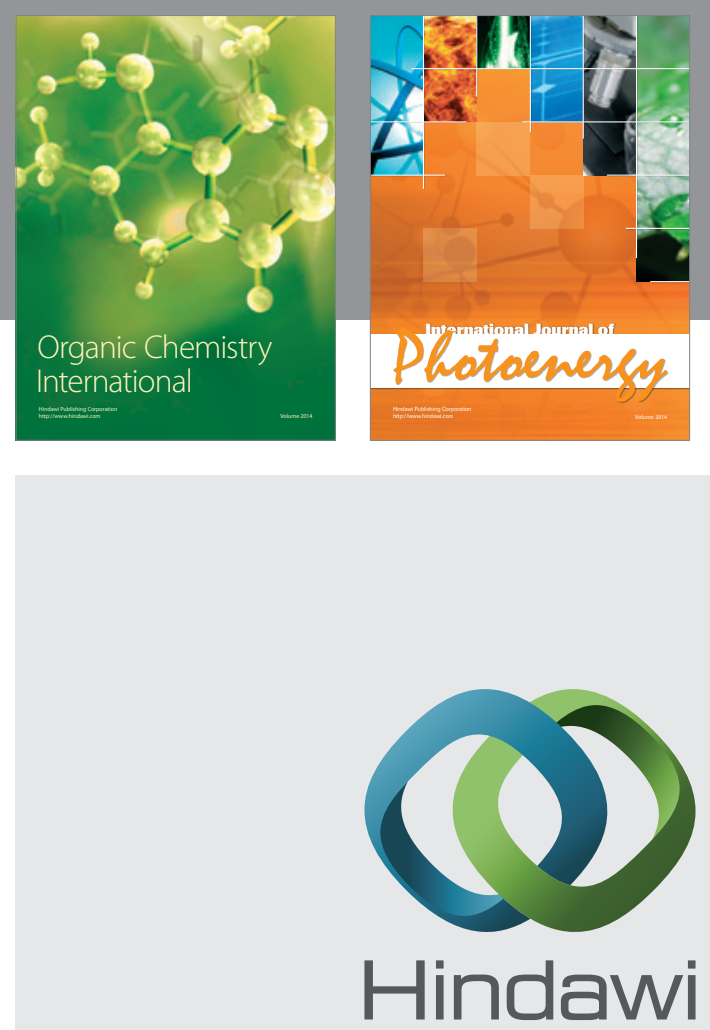

Submit your manuscripts at

http://www.hindawi.com
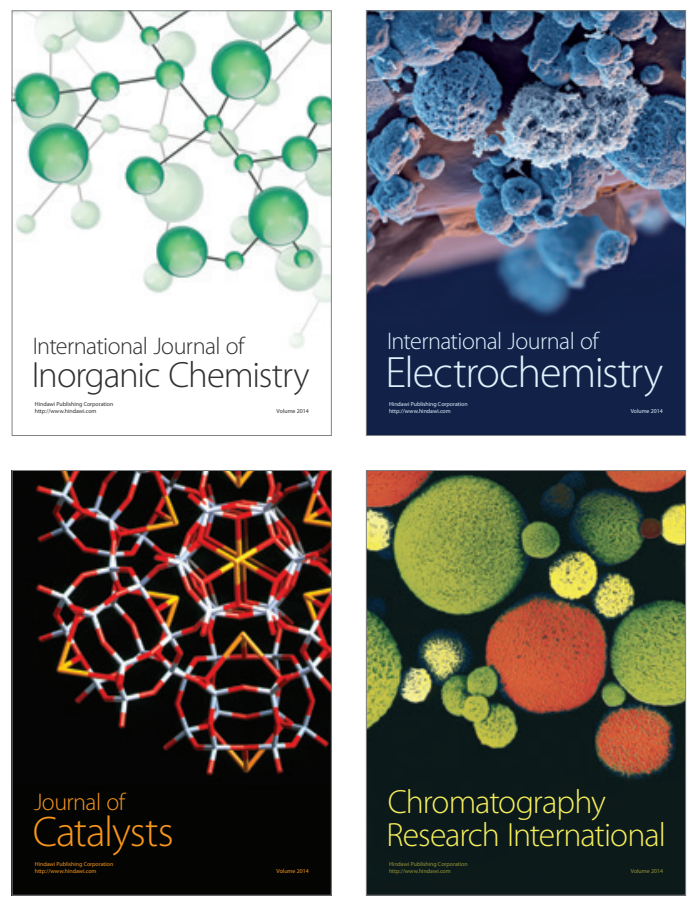
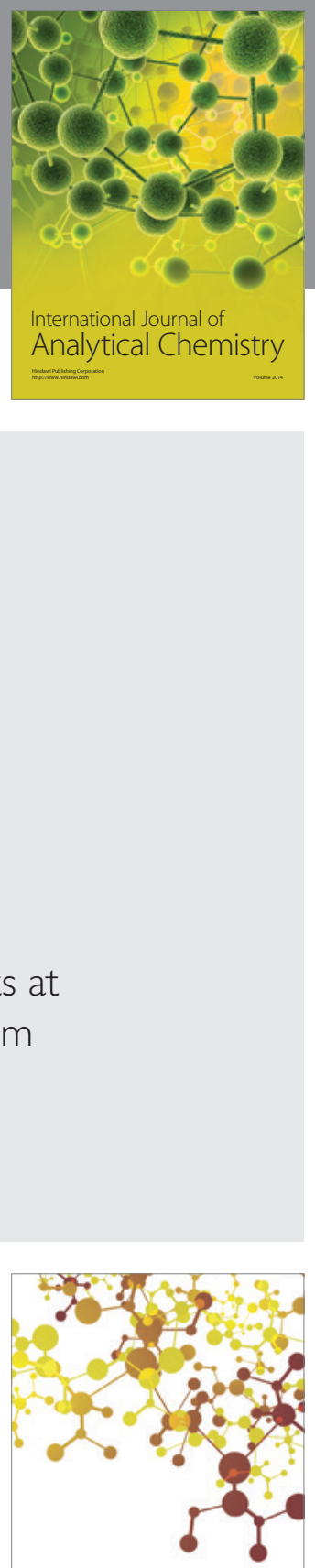

Journal of

Applied Chemistry
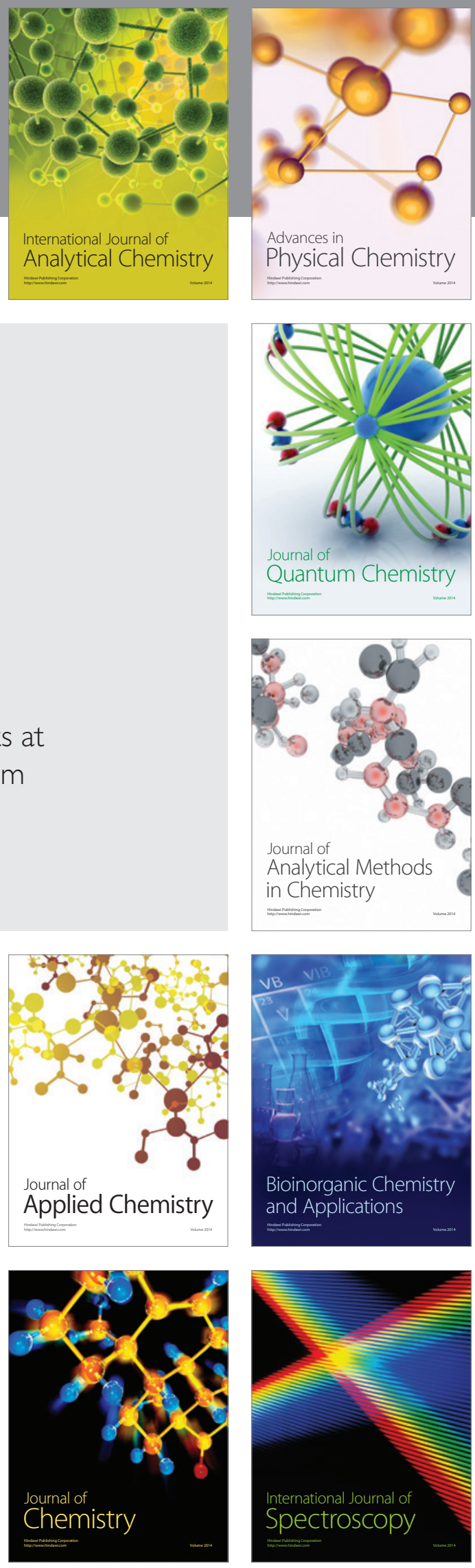